\title{
Images Big and Soft: The Digital Archive Rendered \\ Cinematic
}

Holly Willis

DOI:10.15664/fcj.v19i0.2379

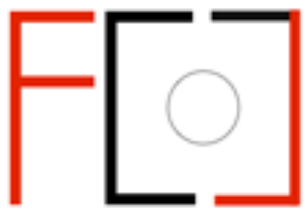

Frames Cinema Journal

ISSN 2053-8812

Issue 19 (March 2022)

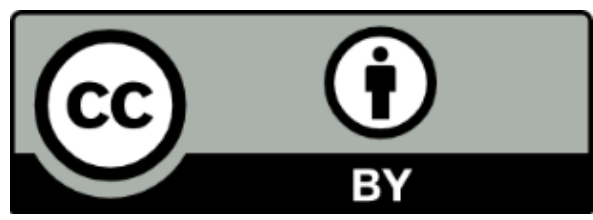




\section{Images Big and Soft: The Digital Archive Rendered Cinematic}

Holly Willis

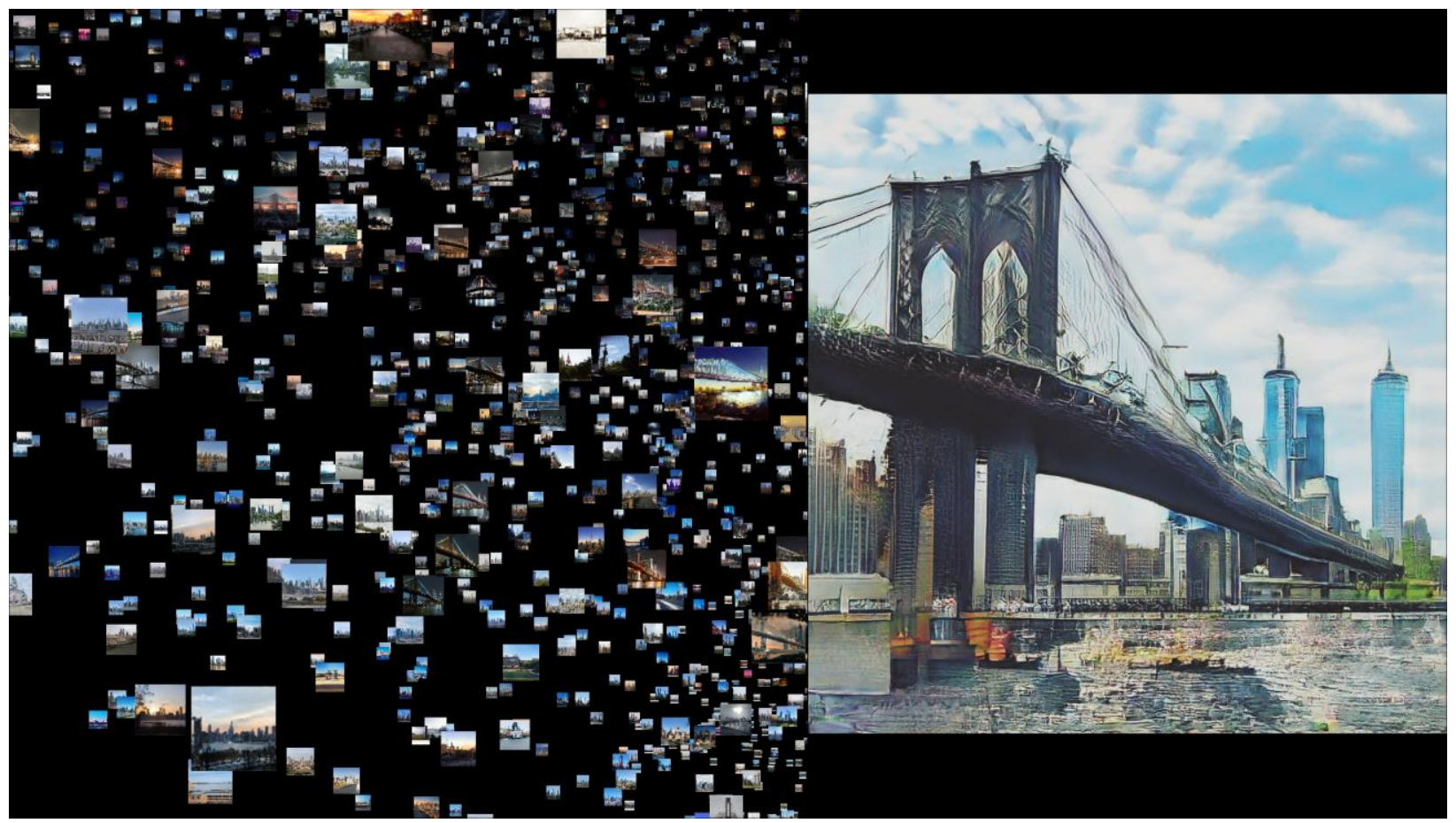

Figure 1: Media artist Refik Anadol uses a tool called the Latent Space Browser developed by his studio to apply machine learning to millions of images to observe a "latent cinematic dimension." These are images created for Machine Hallucination (2021).

\section{Introduction}

In his recent project titled Machine Hallucination (2021), Los Angeles-based media artist Refik Anadol collected over 100 million images of New York City from social media and, using machine learning to "read" the images, created a 30-minute immersive experimental cinema experience that visualises the archive of snapshots. On his website, Anadol explains that computation allows "a novel form of synesthetic storytelling through its multilayered manipulation of a vast visual archive beyond the conventional limits of the camera and the existing cinematographic techniques."1 
In a somewhat similar gesture, in 2018, while participating in an artist's residency in the Netherlands, artist/researcher Anna Ridler took 10,000 digital pictures of tulips, each flower centred against a black backdrop and dutifully labelled by hand. The images were gathered with an intention to demonstrate the labour, ethics, and skill associated with assembling a dataset that could be used for machine learning. The flower images became the framework for a suite of media art projects, each offering a reflection on the shifting nature of the archive with an emphasis on an in-between state, one that brings into the foreground what is so often eclipsed in the collection of data.

In yet another archive-oriented work employing computation, in a project titled Ich halte es für eine Tragödie, daß wir uns nicht gefunden haben! [I consider it a tragedy that we have not found each other!] (2016), media artist Ornella Fieres transformed a box of letters and images found at an inheritance sale, simultaneously reading and reimagining the materials through machine learning. The result is a series of almost uncanny images and text fragments that suggest not simply the as-yet imperfect rendering capacities of machine learning, but rather the potential for an aesthetic based not on mimicry but on failure.

In this essay, I argue that, while these three projects work toward disparate ends and function at radically different scales, from the massive to the intimately personal, taken together, they represent a shift in our understanding of both the cinematic moving image and the archive, and enact a set of new relationships between human vision and digital images. They showcase the image not as stable representation but as unfolding and ongoing process, and they call attention to the fact that the perspective made available to the human is but one among many possible points of view. I should note that I use the word "archive" here in a deliberately broad sense. 
While in the past archives denoted collections devoted to the conservation and creation of historical record based on artefacts, with digital media, notions of image collections, datasets, and archives begin to blur. For my purposes, then, the "archives" constituted by these artists are also datasets and image collections; all three meanings cohere, in part specifically to unsettle the traditional understanding of the archive itself. ${ }^{2}$ I will note also that my emphasis on the unsettling of stability is aligned with scholars who attend to computation and technical conditions, rather than those who describe the "anarchive" as a conceptual reorientation. ${ }^{3}$

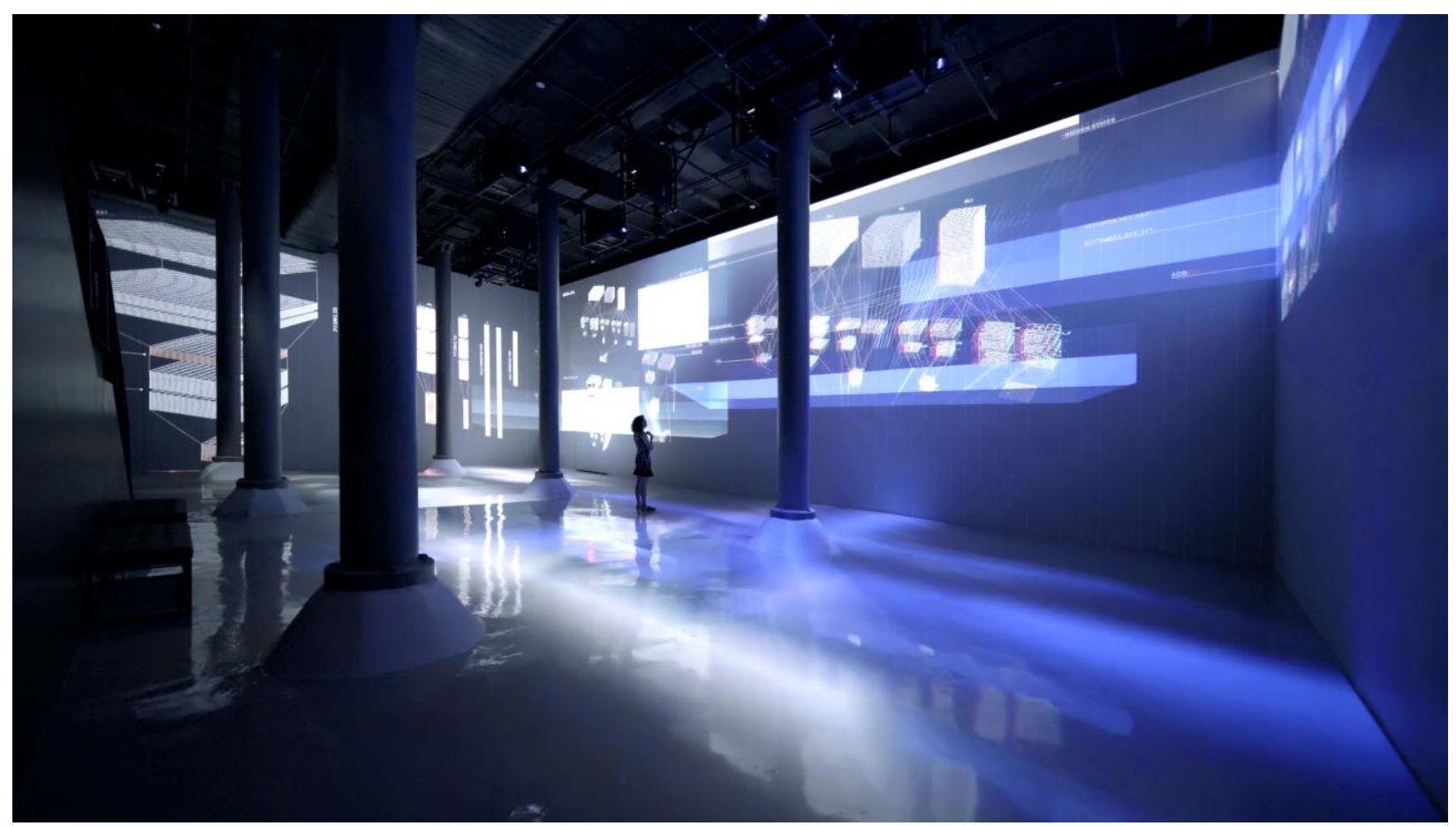

Figure 2: Machine Hallucination (2021), Refik Anadol.

Whereas in the past, the moving image and the archive relied on notions of linearity, organisation, stability, and a semblance of order, the digital archive as it is differently enacted in these three projects suggests contingency, permeability, and process. True, elements of the traditional archive remain in the sense that each of the projects involves collection, ordering, observing connections, and presenting forms of access. However, rather than assuring a history 
or preserving the past, the archive modelled by these projects is in flux, and its qualities are perhaps more correctly aligned with instantaneity. I make this argument not in order to bemoan the loss of stability in a world rife with electronic networks, but instead to suggest that this experience of instability is itself based on the visual, and through that, on a potentially obsolescent understanding of the image within the era of computation. To borrow a phrase from Daniel Rubinstein and Andy Fisher, the photograph that exists within a database is a skeuomorph, an object whose appearance masks its true makeup. In this case, it suggests the innocuous snapshot of the past while in fact functioning far more powerfully within structures of surveillance, control, and power. ${ }^{4}$

Indeed, the projects I have selected are significant in their evocation of what has been named by Ingrid Hoelzl the "soft-image" or "post-image," shifting from the single image as a solid, stable representation within a collection of similarly single images, to that of the distributed, in-process experiential image. ${ }^{5}$ Further, each artist discussed here approaches the creation of a collection of images with varied intentions that in turn illustrate a different facet of the post-image. Each also presents the material in disparate modalities that, while connected to the cinematic, produce disparate sensory experiences that point toward the post-cinematic. Most significantly, taken together, these three artworks offer a perspective on the archive in 2022 and reflect our current moment's transition from representation to computation, as well as an experience of the archive that posits new sensorial experiences that limn the boundaries of the cinematic.

\section{Artists and Algorithms: Technical Notes}

Refik Anadol, Anna Ridler, and Ornella Fieres are just three artists are among many who have begun to explore the efficacy of what is known as Generative Adversarial Networks (GANs) in 
relation to moving image artwork. I highlight this point to point to the contributions artists often make in the evolution of new technologies. In summary, the GAN process generally involves two steps: in the first stage, a computational model is trained to "understand" a set of images by being "fed" a large number of reference images from a particular film or group of films; in the second step, the computer generates images with the model that are in some way related to the original images. Artists may then use the resulting material in a variety of ways.

For scholars, filmmakers, and artists unfamiliar with the brief history of the use of Generative Adversarial Networks in filmmaking, I offer a more detailed overview of the technical language and processes associated with them. Machine learning is a term developed by Arthur Samuel in the 1950s based on a program he created that learned how to play checkers. As the program played the relatively simple game, it developed improved skills, gradually surpassing the ability of human players. Machine learning, then, is a part of the larger field of artificial intelligence and describes the process through which data and algorithms emerge not simply as "recipes" that dictate a set of actions, but instead allow the machine to learn over time, based on a process of "training" using existing data. When we use Netflix, for example, the platform tracks our viewing habits and looks for connections and patterns across genres, directors, performers, and so on, and then recommends other films where it finds similarities or connections. The algorithm trains itself based on the information it gathers from users; the more data it collects, the more accurate and more effective it becomes over time. ${ }^{6}$

Generative adversarial networks, which have grown more sophisticated over the last five years, take machine learning a step farther. In this process, a generator network offers random image samples to what is known as a discriminator network, which in turn attempts to ascertain which 
images are real and which are fake. The generator grows better over time at creating images that are real enough to fool the discriminator; the back-and-forth process creates a powerful learning system.

A group of artists have been interested in the development of machine learning specifically in the context of cinema. For example, artist and researcher Terence Broad has explored the use of GANs, and, in an essay describing his process, explains his attempts to use GANs to create more realistic images. He sketches a history, noting that the adversarial process was initially developed by Ian J. Goodfellow and his colleagues in 2013, but it was not until two years later that the process was able to produce realistic images. ${ }^{7}$ Building on the work of Goodfellow and Alex Radford, Broad tried to design a variational autoencoder in which the discriminator network could "assess how similar a reconstructed sample is to the real sample." 8 This would allow the GAN to achieve precision more quickly. He explains that before he was able to create this adjustment, Anders Boesen Lindbo Larsen published a paper that moved the process forward. ${ }^{9}$ In this method, the system compares "the difference in response of the real and reconstructed samples in the higher layers of a discriminator network" that creates a "learned similarity metric" that is not focused on a pixel-based reconstruction error comparison. ${ }^{10} \mathrm{He}$ goes on to explain that Larsen's model relies on an encoder, decoder, and discriminator.

Broad used this method to work with Ridley Scott's Blade Runner (1982), the experimental feature film Koyaanisqatsi (1982), and John Whitney's Matrix III (1972), creating what he calls "reconstructed films." While it is fascinating to see how the algorithm tries to reproduce the faces of actors, scenes from nature, and computer-generated animation, the most interesting experiment conducted by Broad centres on Richard Linklater's 2006 film A Scanner Darkly 
(2006). Linklater shot this project on film, and then the live action footage was transformed using software that automated the rotoscoping process to create an animated feature. In the sideby-side comparison of the film's trailer and the autoencoded version of the trailer, the transformed version boasts a painterly blur and wash of colour absent in the original. In a sense, the new version of the trailer is somehow more animated.

As another example of artists involved in using GANs in relation to cinema, Casey Reas wrote a book titled Making Pictures With Generative Adversarial Networks (2019. In it, he describes his process, writing specifically from the perspective of an artist rather than a computer scientist. He explains, "A GAN model generates pictures by inputting a list of one hundred numbers between -1 and 1 . For instance, if all one hundred numbers are set to 0 , a specific picture will be produced that correlates to those values. If the first number is changed to 0.1 , a similar but different picture will be generated." 12 Like Broad, Reas has used GANs to visually reimagine existing films. For example, he trained a DCGAN model on frames from Ingmar Bergman's 1966 film Persona. He notes that some of the images produced in this process resemble the film's actors; others are odd hybrids of landscapes and bodies; and others are beautiful abstractions. Highlighting the images' uncanniness, Reas writes: "A subset of images created through the GAN are an alternate way to imagine this essential aspect of the film."13

In a final example, a research team composed of Anirudhan Iyengar, Yulia Marouda, and Hesham Hattab at the Interactive Architecture Lab at the Bartlett School of Architecture at University College London created a project called "Neural Kubrick" as Iyengar's thesis project, with a very different agenda than that of Reas. The goal was to consider how AI might be used within the filmmaking process itself, focusing on 2001: A Space Odyssey (1968), A Clockwork 
Orange (1971), and The Shining (1980). In an interview, Iyengar describes his desire to see if three different machine learning algorithms could tackle three key aspects of the filmmaking process - namely, art direction, editing, and cinematography - by using a dataset of 115,000 frames drawn from 100 movies. As he explains:

There is a Generative Adversarial Network (GAN) that reimagines new cinematic compositions, based on the features it interprets from the input dataset of movie frames. There is a Convolutional Neural Network (CNN) that classifies visual similarities.... And there is a Recurrent Neural Network (RNN) that analyses the camera path coordinates of a cinematic sequence and generates new camera paths to reshoot the original input sequence in virtual space.... ${ }^{14}$

While it is difficult to interpret the results of the project based on the three short videos that demonstrate the machine as art director, editor, and cinematographer, the shared impulse to collaborate with machine learning to reimagine the capacities of the moving image point to a larger cultural curiosity about the connections that might be made across extensive collections of images.

In each of these cases, artists have employed machine learning to explore cinema, in effect imagining cinema not as the linear unfolding of moving images but instead as a dataset or archive with which to experiment. This reorientation, from the representation of a film on screen to the collection of a group of images to explore, enacts the shift from cinema as story to cinema as database ripe for computational manipulation. 


\section{Machine Hallucination}

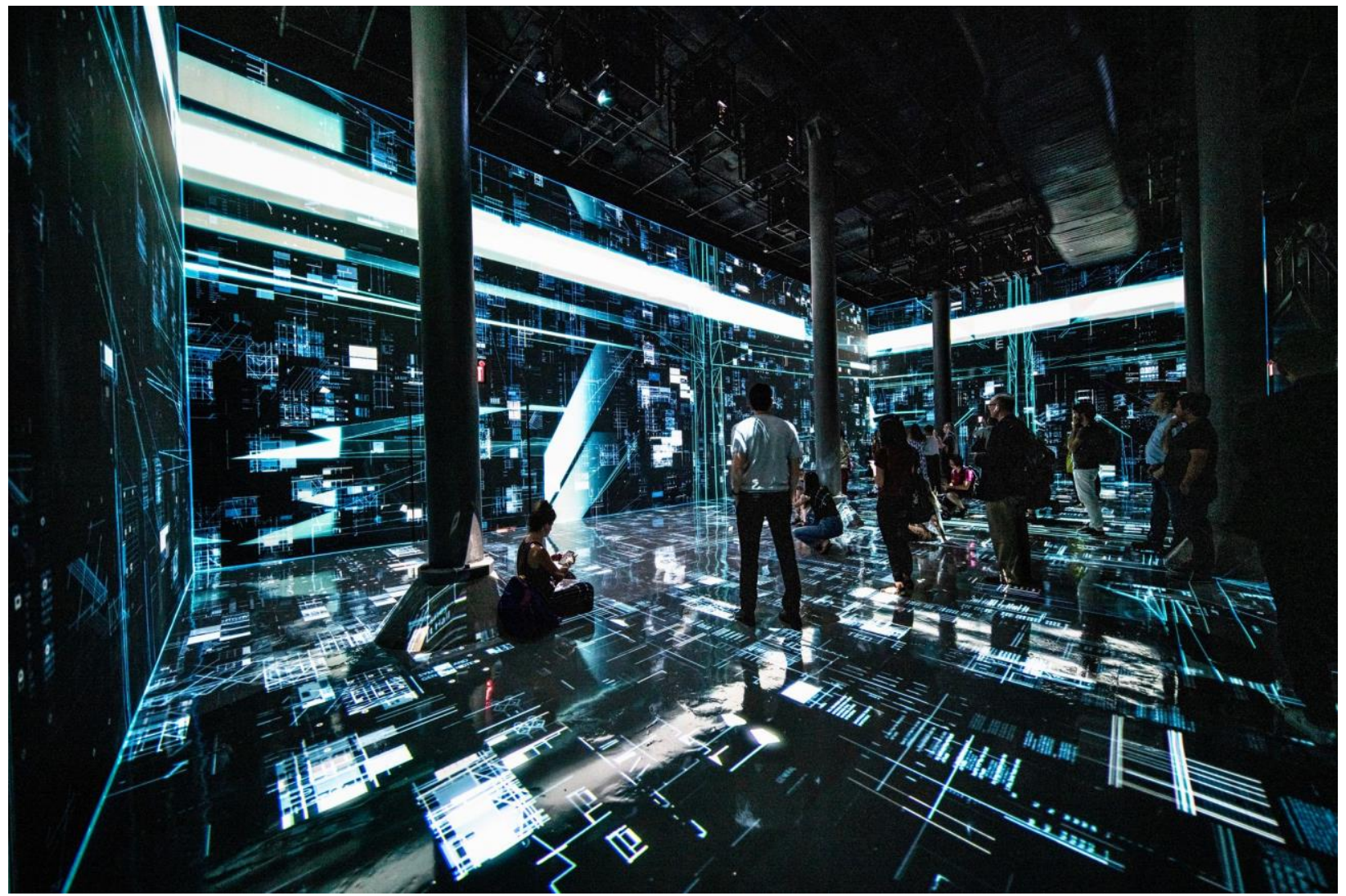

Figure 3: Machine Hallucination (2021), Refik Anadol.

Turkish artist Refik Anadol has long been fascinated by the intersection of computation and cinema and he is known for his large-scale public urban artworks that typically integrate and visualise data, creating a sense of correspondence between the often-invisible forces around us and our own lived experience. The works tend to borrow the scale and visual power of cinema; they are grand, extraordinarily beautiful, and captivating. As his work has developed over the last decade and his use of computation has grown more extensive, the scale of the work has also increased.

This is evident in Machine Hallucination, with its millions of photographs of New York City, culled from various social networks and fed into a GAN. By applying machine learning to the images, Anadol and his team shift attention from the traditional modes of photography and 
filmmaking which focus on the capture, processing, editing, and exhibition of images, instead foregrounding the collection, collation, and layering of images. The result is not an image but a phenomenon known as the post-image. ${ }^{15}$

Designed for Artechouse, a space designed to showcase media art in New York, the project is presented as a large-scale projection of a 30-minute video in $16 \mathrm{~K}$ resolution that moves through three specific chapters. The installation makes use of multiple projectors to create an immersive moving image experience that fills the walls and floor of the gallery. The imagery is dazzling as thousands of shapes - suggesting individual images from the archive - swirl and dance. At times the images are presented in a grid; some showcase the animated GANs as compilations of images are layered together. As the experience grows increasingly more dramatic, the images become wave-like, resembling colourful breakers, roiling and crashing, moving from greens and blues to reds and oranges. The viewer stands in the midst of this visual cacophony, sensing the overwhelming proliferation of images. Indeed, Anadol's work recalls a set of terms used to describe film and digital media at various points over the last century, as when Scott Bukatman writes of "technological spectacle" and "kaleidoscopic perception" in relation to media forms that "invoke heightened, even exaggerated, bodily awareness in relation to highly technologized environments" in his 2003 book Matters of Gravity: Special Effects and Supermen in the $20^{\text {th }}$ Century. ${ }^{16}$ While Bukatman discusses the sense of frenetic delirium coextensive with urban modernity of the last century, Anadol gives us the experience of the image removed from its representational role and let loose to function not only through radical mobility and kineticism, but as an immersive world - the data stream we hear of so often made manifest. 


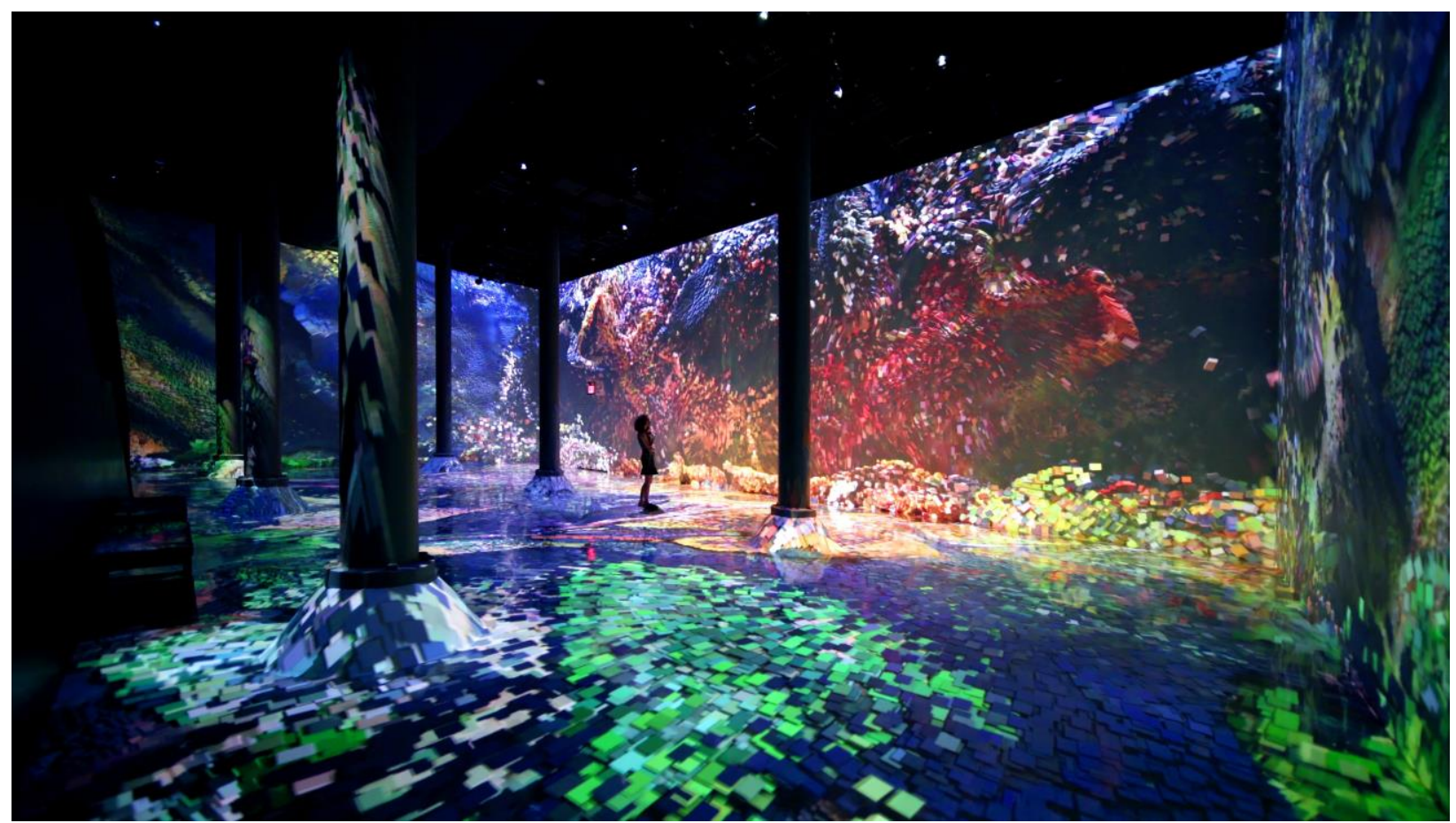

Figure 4: Machine Hallucination (2021), Refik Anadol.

Anadol's dizzying experiences of the archive overwhelm and confuse; they remap the relationship between human and machine; they offer new experiences of space and time; the orientation of the body and the gaze in cinema here is replaced and the body is disoriented. We might initially attribute this confusion to a shift from narrative, so fundamental to notions of cinema, to the archive. As Ernst van Alphen argues, "[w] hereas the role of narrative is in decline, the role of archive, in a variety of forms, is increasing." ${ }^{17}$ He goes on to echo Lev Manovich and the claim that the database has become the dominant symbolic and cultural form. ${ }^{18}$ With the waning of narrative we see, too, the dissolution of the structuring principles of story. Van Alphen continues: "[a]s a result of this cultural change the symbolic form of (syntagmatic) narrativity has a more modest role to play. It is no longer the encompassing framework in which all kinds of information is embedded, but the other way around. It is in the encompassing framework of archival organizations that (small) narratives are embedded." ${ }^{19}$ Van Alphen and Manovich both point to the cultural shift from narrative to database with attention specifically to narrative 
structures. The linear timeline of film gives way to the display of innumerable image and narrative choices, and celebrates nonlinearity, looping structures, and circularity. Where traditional storytelling, especially in classical narrative cinema, privileges causal unfolding and thematic coherence across time, database structures call forward alternative structures borrowed from the world of computation.

However, it is not simply the displacement of narrative that produces the dizzying sensation. Indeed, this sense of confusion also characterises many recent Hollywood feature films, a fact addressed by a number of writers in relation to disruptions in continuity editing and a resulting lack of spatial and temporal coherence in digital cinema and what has now been dubbed "postcinema" by scholars Steven Shaviro, Shane Denson, Julia Leyda, among others. ${ }^{20}$ Cameras become virtual and free-floating, no longer tethered to a perceiving body, and narratives become convoluted, resembling game structures, loops, and puzzles, not only in their challenging structures, but in their pleasures, shifting from narrative immersion to problem-solving, repetition and pattern recognition. ${ }^{21}$

We might also attribute the sense of disorientation to the somatic immersion of Anadol's project. Janet Murray, writing more than 20 years ago in Hamlet on the Holodeck (1997), explores immersion in relation to its fundamental role in new media. She explains that we "seek the same feeling from a psychologically immersive experience that we do from a plunge in the ocean or swimming pool: the sensation of being surrounded by a completely other reality, as different as water is from air, that takes over all of our attention, our whole perceptual apparatus." ${ }^{22}$ Murray's description captures the sense of fluidity in the cascade of images, with its swirling patterns, scale, and continual motion. 
Given a cultural wariness toward the increasing sense of surveillance connoted by digital media, we may feel inclined to cast suspicion on the artist who turns images into data, seemingly using the still images as fodder to create waves of visual spectacle. However, we need to know that the images had no original pure state. They were not unique and pristine pictures that were then dissolved into the swirling stew of an immersive experience. Instead, they began as noise, perhaps were tuned toward signal, and then, with Anadol, returned to noise (albeit beautiful noise). Indeed, a computational understanding of photography offers a very different model of the visual, shifting from still image to image as process, from a picture taken to a picture produced. Ingrid Hoelzl explores this concept in her essay "Image-Transaction" (2020), in which she outlines a view of images that stems from a description of the relationship between humans and their milieu presented by pragmatist philosopher John Dewey and sociologist Arthur Bentley in their 1949 book Knowing and the Known, which they described as "transactional." ${ }^{23}$ Hoelzl extends Dewey and Bentley's understanding of the entanglement of humans and world to include images, noting that she is moving beyond simply understanding the processual nature of the digital image. She describes the digital image in this way:

Image and data, screen and network are in fact part of a transactional ensemble where (when) the image, as the visible part of a given data exchange, coincides with the screen, as the local access point to the network - access understood as both a functional capacity and a process. Images are not merely outputs displaying network process on an outpost (the screen as network terminal). Indeed they stand (or rather proceed) in continuous relation with server and client computers, data and algorithms, signals and sensors). ${ }^{24}$

In a sense, then, we might understand Anadol's Machine Hallucination as an enactment of the radical destabilisation of our concept of the image. While we tend to hang on to the idea that an image, even one on-screen, is a simple picture, it is in fact an entity that is processual, 
Frames Cinema Journal, Issue 19 (March 2022)

transactional, and surveillant. The hallucination, then, in Anadol's project is perhaps more aptly attributable to the human. These images are often presented in a state of perpetual becoming from which it is impossible to extract a single, static, definitive frame; they are always flowing, morphing, and evolving without boundaries or fixed edges. In this regard, the comparisons with artifacts of human cognitive process seem apt, reflecting the slippage with which we recall memories, dreams, or hallucinations. Nevertheless, these metaphors for consciousness disserve and misdirect our understanding of their computational and algorithmic origins.

\section{Tulips in the Algorithm}

London-based British artist Anna Ridler has created a suite of projects that reflect on the nature of the database. Images of Ridler's work may be viewed on her website, http://annaridler.com/. Myriad (Tulips) (2018) is a collection of ten thousand C-type digital prints annotated by hand and displayed in a grid formation on the wall of galleries and museums with magnets. Created while the artist was engaged in a residency in the Netherlands, the pictures were intended to bring forward some of the questions and issues sparked by datasets and machine learning. These include the role of the human in general in creating datasets, and the issue of labour that too often and too easily goes unnoticed or uncompensated. She brings an attention to labour in the project by emphasising her actions in taking each picture, writing on each one, and then mounting them one-by-one on the walls of the gallery for her shows. The introduction of the human - with wavering handwritten words and an inexact eye trying to align the images to create

a grid - reminds us of the efficacies of each modality, the machine with its precision and the human with our fallibility. 
Ridler explains that her decision to use tulips is tied to their connection to notions of speculation within the context of "tulipmania," a moment in the mid-1600s in the Dutch Republic when the popular flower grew fashionable, which in turn drove prices for tulip bulbs exceptionally high before a dropping in a sudden collapse. While much of folklore connoted by tulipmania has been shown to be untrue or exaggerated, as historian Anne Goldgar highlights in her book, Tulipmania: Money, Honor, and Knowledge in the Dutch Golden Age, tulipmania nevertheless "gives us a chance to look in microcosm at a society that was, indeed, grappling with its material values and the relation they bore to their social ones." ${ }^{25}$ In drawing a connection to tulipmania, Ridler suggests a corollary between the boom and bust of the tulip between 1634 and 1637 and the often outrageous claims made today regarding the value and power of NFTs, Silicon Valley startups, algorithms, and computation itself.

Ridler expanded on her collection of tulip photographs in two subsequent video projects titled Mosaic Virus (2018) and Mosaic Virus (2019). Both use the image of tulips as their focus, and both continue the artist's interest in drawing parallels between forms of financial speculation in the past and the present. However, for my purposes, the projects pinpoint the shifting status of the moving image as it engages computation and datasets. Mosaic Virus (2018) features a grid of tulips in bloom on a single screen. However, the images of the tulips shift and change based on the price of bitcoin. Each individual image, then, is no longer a "picture" that belongs to an "archive." Instead, each image becomes a mutable index of value shifts, and the larger grid becomes a register of financial change across multiple inputs.

Mosaic Virus (2019) is a three-screen video installation also showing tulips; in this case, each screen features a single flower. The flowers shift and change in colour, size, and shape, again in 
Frames Cinema Journal, Issue 19 (March 2022)

correspondence with the price of bitcoin. Here, too, the image is no longer a photograph or even a drawing; the image instead serves as the interface between pictorial representation and computation, and as a figure for reminding us of archives of the past. The singular image gives way to the multiple; stasis succumbs to mutation.

In the introduction to their edited collection On the Verge of Photography: Imagining Beyond Representation, co-editors Daniel Rubinstein and Andy Fisher describe the contemporary photograph's role in sustaining a "multi-layered reality" as we move seamlessly among layers of data, imagery, and matter. "It seems that the digital-born image has become a hinge between these physical and digital modes of existence," they write, "combining as it does elements of familiar ocularcentric culture - with its trust and reliance on the true-to-life photograph - and algorithmic processes that problematize the presumption of an ontological connection between images and objects." 26 Ridler's projects bring to the fore the shifting nature of the image, showing us clearly that it is a construct designed specifically to enact its capacities beyond merely the realm of the visual. This is an image that is in a sense inhabited by information and is therefore ongoing and emergent rather than static and historical. As Rubinstein and Fisher note in regard to the temporality of the image, the digital networked image "is not an archive of past events but a force that shapes the present." ${ }^{27}$ Indeed, Ridler fed some of the images into a generative adversarial network (GAN) that she used to create a series of videos, which she then sold in 2019 in an online auction as part of the first wave of NFT art.

\section{An Intimate Archive: Letters and Postcards}

In 2016, German artist Ornella Fieres came across a box of materials that belonged to a woman who had lived in the former GDR, in East Berlin, in the 1960s and 1970s. The box contained 
hundreds of letters, postcards, and images. Fieres has since used this personal collection of materials to think about the ways in which artificial intelligence and computation - the infrastructures that increasingly shape our status as citizens and that scaffold our relationships to each other - might conjure unknown the woman based on a process of sifting through her intimate archive.

Three specific projects have emerged from the artist's interactions with this box of materials. In "Postcards to M," Fieres fed two hundred postcards from the box into a neural network and the AI generated new images of the flowers. They are at once ethereal and almost grotesque in their sense of deformation. Fieres displays the images as large-scale framed prints. The petals are mottled and uneven, and even appear fleshy in places; presented as larger-than-life, the images suggest a celebration of a kind of uncanny monstrosity, referencing both flowers and the human body but resembling neither entirely.

In a second project, titled "Letters to M_HTR," Fieres fed over seven hundred letters from the collection into a network which tried to "read" the handwriting on old, even mouldy paper. Fieres displays the AI's attempts at translation as a series of text fragments, shown in random order on three stacked television sets. The fragments are nonsensical and often amusing, and when they appear in black and white on the old television sets, they flicker, an uncanny hail from the past across multiple technologies to the gallery in the present.

For the third part of the project, Fieres worked with the photographs that were in the box, using artificial intelligence to interpret the images. For the gallery exhibition, Fieres shows the back of the image, along with the text fragment description of the image produced by the AI. "A white 
bird is standing on a ledge," is one example of the text fragment. We do not see the images but instead try to imagine what the computer saw that would render this description.

Fieres has said that her work is concerned with occultism and technology. "I build photographic apparatuses and manipulate algorithms, artificial intelligence, or software to create images that carry traces of the past and might be a foreshadowing of events in the future. ${ }^{28}$ Indeed, Fieres deftly sketches a spectrum of times and technologies in these pieces, linking forms prominent in the past - letters and postcards, static images, and televisions - to present technologies of machine learning and image synthesis. Seeing the odd distortions, we at once acknowledge the limitations of the technology and its seeming inability either to render a decent image of a flower or to read handwriting, but also revel in the uncanniness of the results. We become aware of another intelligence at work, and while we cannot see it, we can sense it.

It is not insignificant that both Ridler and Fieres have chosen flowers as their subject matter for their projects. Flowers are rich metaphors for the precarity of life, as well as exemplars of the ways in which the so-called natural world is now readily industrialised and produced. Flowers, however, retain their power of allegory and representation of beauty. Offering delicate flowers into the number-crunching machine of the computer brings to the fore the symbolic violence of computation, as well as the literal environmental costs of computing in the context of climate collapse. It also serves to distinguish the characteristics of both. In her book From Point to Pixel: A Genealogy of Digital Aesthetics, Meredith Hoy references David Summers and the notion of universal metric space from his book Real Spaces: World Art History and the Rise of Western Modernism (2003). She explains that Summers develops a description of the features that make up "universal metric space" and its attendant qualities of "homogeneity, divisibility, and infinite" 
that she says "stand in contrast to the 'qualitative, continuous, and unified' spaces associated with the natural, primordial world." 29 She continues: "[o]ne of the primary characteristics of the transition between qualitative and quantitative systems of measurement is that while qualitative systems retain some reference to the specific qualities of the particular, situated, and enworlded object subject to measurement, purely quantitative systems are notional, abstract, and separable from the format (the size, shape, volume and texture of the surface of inscription) on which an image is presented." ${ }^{30}$ Reading this in conjunction with the projects of Riddler and Fieres, we can see how both stage a confrontation between these two spaces; the flower, in its particularity and situatedness becomes abstract and notional, which is further represented in the fact that, in Ridler's work, it becomes animated.

A final point contributes to my broader argument: the imagery presented in these artworks hovers somewhere between photograph and illustration, between live action and animation, and indeed, the categories begin to lose their valence altogether. While in each case, the images are rooted in history in some manner, they lose their historical status in their lack of indexical legibility. In this way, the projects serve yet another function in this transitional moment. I turn to James J. Hodge to address this shift. In his recent book on contemporary digital media, Hodge argues that animation in particular offers a modality best suited to expressing contemporary historical experience. ${ }^{31}$ His argument is that language-based narrative representation was the modality deployed to write history in a previous century, while animation steps forward as the appropriate form for a new era. "Animation allows for phenomenal encounters with the experiential opacity of digital media precisely without dispelling that opacity," he writes, adding that, "[a]s a field of aesthetic forms based on the perception of absent causes, animation instantiates the very character of a digital output whose origin is always and fundamentally ever 
hidden from view." ${ }^{32}$ Hodge is referring to the ways in which computation remains invisible to us; we see only its effects. Similarly, animation obscures its substrate. He explains that animation points to what he calls the "experiential opacity of digital media," and further, it captures its "time-based volatility."

Taken together, the artworks by Anadol, Ridler, and Fieres explicitly engage with the proliferation of data in the $21^{\text {st }}$ century, and indeed, as computational artworks, are uniquely able to draw connections between contemporary experiences of the visible and invisible, skeuomorph and data. The three projects in a sense offer instruction in how to rethink the cinematic image, not as a complete and coherent entity but as archive-in-process, continually transforming, mutating, and shifting perspective, not as human-oriented point-of-view but as fluid morph with its own machinic sense of time and space.

\section{Notes}

${ }^{1}$ Refik Anadol, https://refikanadol.com/works/machine-hallucination/

${ }^{2}$ David M. Berry addresses this shift in his essay "The Post-Archival Constellation: The Archive Under the Technical Conditions of Computational Media" He writes, "Computation therefore threatens to dearchive the archive, disintermediating the memory institutions and undermining the curatorial functions associated with archives." In Memory in Motion: Archives, Technology and the Social, Ina Blom, Trond Lundeno and Eivind Røssaak (Amsterdam: Amsterdam University Press, 2016) 117.

${ }^{3}$ See, for example, The Go-To How To Book of Anarchiving (2016) created at Concordia University's Senselab; it describes the anarchive as "an excess energy of the archive," as the archive's supplement, and as a technique "for making research-creation and process-making engine." Brian Massumi, "Working Principles," The Go-To How To Book of Anarchiving (Montreal: The Senselab, 2016), 7. http://senselab.ca/wp2/immediations/upcoming-distributing-the-insensible-dec-10-20-2016/the-go-tohow-to-guide-to-anarchiving/. My rethinking of the archive is also very different from the questions oriented toward the power and exclusions of the archive. See, for example, Mandeeq Mohamed's "Somehow I Found You: On Black Archival Practices," in which the author notes, "I want to look at black lives as the excess of the archive, the messiness of histories that cannot be so easily recorded and understood, simply because there is far too much at stake to ever assign anything like humanity to black life.” In C Magazine, Issue 137, Spring 2018, np.

${ }^{4}$ In the introduction to their book, Daniel Rubinstein and Andy Fisher write, "[i]n contrast to earlier forms of photography, the digital-born image seems defined by how it exceeds familiar terms of visual experience. What one sees as an image on-screen for instance, is only conventionally presented to appear the same as the analogue photograph: it is actually a skeuomorph." They go on to explain that the image 
is in fact "a variegated field of data that is not bound to obey the material and visual logic often take to be defining of photography." "Introduction," On the Verge of Photography: Imagining Beyond Representation, edited by Rubinstein, Fisher and Johnny Golding (Birmingham, UK: ARTicle Press, 2013), 11-12.

${ }^{5}$ See Ingrid Hoelzl and Rémi Marie's Softimage: Towards a new Theory of the Digital Image (Bristol, UK: Intellect Books, 2015).

${ }^{6}$ See Sara Brown's very clear overview of machine learning in "Machine Learning, Explained," MIT Sloane School of Management, Ideas Made to Matter, April 21, 2021. https://mitsloan.mit.edu/ideasmade-to-matter/machine-learning-explained

7 "Autoencoding Blade Runner: Reconstructing Films With Artificial Neural Networks," Medium, May 24, 2016. Broad references the research article "Generative Adversarial Nets" by Ian J. Goodfellow, Jean Pouget-Abadie, Mehdi Mirza, Bing Xu, David Warde-Farley, Sherjil Ozair, Aaron Courvell and Yoshua Bengio, University of Montreal, 2014.

${ }^{8}$ Broad, "Autoencoding Blade Runner," np. Broad is referencing the research paper titled "Unsupervised Representation Learning with Deep Convoluted Adversarial Networks" by Alex Radford, Luke Metz and Soumith Chintala, 2015.

${ }^{9}$ Anders Boesen Lindbo Larsen, Søren Kaae Sønderby, Hugo Larochelle, and Ole Winther,

"Autoencoding Beyond Pixels Using a Learned Similarity Metric," 2015.

${ }^{10}$ Broad, "Autoencoding Blade Runner," np.

${ }^{11}$ Broad, "Autoencoding Blade Runner," np.

${ }^{12}$ Casey Reas, Making Pictures With Generative Adversarial Networks (Montreal: Anteism Books, 2019) 17.

${ }^{13}$ Reas, 20.

${ }^{14}$ Luke Dormehl, "See What Happens When AI Tries to Reimagine Stanley Kubrick's Films," Digital Trends, November 23, 2017, no page number. https://www.digitaltrends.com/cool-tech/neural-kubrickproject/ See Iyengar's personal website as well: https://www.anirudhaniyengar.com/neuralkubrick

${ }^{15}$ See Softimage: Towards a New Theory of the Digital Image, Ingrid Hoelzl and Remi Marie (Bristol, UK: Intellect, 2015); Fragmentation of the Photographic Image in the Digital Age, Daniel Rubinstein, ed. (New York, NY: Routledge, 2020); and Discorrelated Images, Shane Denson (Raleigh, NC: Duke University Press, 2020).

${ }^{16}$ Scott Bukatman, Matters of Gravity: Special Effects and Supermen in the $20^{\text {th }}$ Century (Durham, NC: Duke University Press, 2003) 2.

${ }^{17}$ Ernst van Alphen, Staging the Archive: Art and Photography in the Age of New Media (London: Reaktion Books, 2014), 7.

${ }^{18}$ Ernst van Alphen, Staging the Archive, 7.

${ }^{19}$ Van Alphen, 12.

${ }^{20}$ See, for example, Post-Cinematic Affect, Steven Shaviro (Washington, DC: O Books, 2010); PostCinema: Theorizing $21^{\text {st }}$ Century Film, Shane Denson and Julia Leyda, co-editors (Palo Alto, CA: Stanford University Press, 2016); The State of Postcinema: Tracing the Moving Image in the State of Digital Dissemination, Malte Hagener, Vinzenz Hediger, and Alena Strohmaier, eds. (London: Palgrave Macmillan, 2016); Post-cinema: Cinema in the Post-art Era, José Moure and Dominique Chateau, coeditors (Amsterdam: Amsterdam University Press, 2020); and Discorrelated Images, Shane Denson (Durham, NC: Duke University Press, 2020).

${ }^{21}$ See Warren Buckland's edited collection of essays titled Puzzle Films: Complex Storytelling in Contemporary Cinema (West Sussex, UK: Wiley-Blackwell, 2009), and especially Thomas Elsaesser's "The Mind-Game Film."

${ }^{22}$ Janet Murray, Hamlet on the Holodeck: The Future of Narrative in Cyberspace (Cambridge, MA: The MIT Press, 1997; updated and reissued 2016) 98-99. See also "Immersivity: An Interdisciplinary Approach to Spaces of Immersion" for an interdisciplinary approach to the concepts of immersion and immersivity. By Florian Freitag, Céline Molter, Laura Katharina Mücke, Helena Rapp, Damien B. 
Schlarb, Elisabeth Sommerlad, Clemens Spahr, and Dominic Zerhoch, Ambiances: International Journal of Sensory Environment, Architecture and Urban Space, 2020.

https://journals.openedition.org/ambiances/3233

${ }^{23}$ Ingrid Hoelzl, "Image-Transaction," in Parallax, 2020, Vol. 26, No. 1, 20-33, Networked Liminality.

${ }^{24}$ Hoelzl, "Image-Transaction," 24.

${ }^{25}$ Anne Goldgar, Tulipmania: Money, Honor, and Knowledge in the Dutch Golden Age (Chicago, IL: The University of Chicago Press, 2017) 18.

${ }^{26}$ Rubinstein and Fisher, eds., On the Verge of Photography: Imagining Beyond Representation, 8.

${ }^{27}$ Rubeinstein and Fisher, 10.

${ }^{28}$ Ornella Fieres, "Artist Statement," in the artist's Portfolio 2021.

${ }^{29}$ Meredith Hoy, From Point to Pixel: A Genealogy of Digital Aesthetics (Hanover, NH: Dartmouth College Press, 2017) 114.

${ }^{30}$ Hoy, From Point to Pixel, 114.

${ }^{31}$ James J. Hodge, Sensations of History: Animation and New Media Art (Minneapolis, MN: University of Minnesota Press, 2019).

${ }^{32}$ Hodge, 15-16.

${ }^{33}$ Hodge, 16.

\section{Bibliography}

Alphen, Ernst van, Staging the Archive: Art and Photography in the Age of New Media. London: Reaktion Books, 2014.

Blom, Ina, Trond Lundeno and Eivind Røssaak. Memory in Motion: Archives, Technology and the Social. Amsterdam: Amsterdam University Press, 2016.

Broad, Terence. “Autoencoding Blade Runner: Reconstructing Films With Artificial Neural Networks.” Medium, May 24, 2016. https://medium.com/@ terencebroad/autoencodingblade-runner-88941213abbe

Brown, Sara. “Machine Learning, Explained.” MIT Sloane School of Management, Ideas Made to Matter, April 21, 2021. https://mitsloan.mit.edu/ideas-made-to-matter/machinelearning-explained

Buckland, Warren, ed. Films: Complex Storytelling in Contemporary Cinema. West Sussex, UK: Wiley-Blackwell, 2009. 
Bukatman, Scott. Matters of Gravity: Special Effects and Supermen in the $20^{\text {th }}$ Century. Durham, NC: Duke University Press, 2003.

Denson, Shane. Discorrelated Images. Raleigh, NC: Duke University Press, 2020.

Dormehl, Luke. "See What Happens When AI Tries to Reimagine Stanley Kubrick's Films." Digital Trends, November 23, 2017, no page number. https://www.digitaltrends.com/cool-tech/neural-kubrick-project/

Fieres, Ornella. “Artist Statement.” In the artist's Portfolio 2021: https://ornellafieres.com/ Freitag, Florian, Céline Molter, Laura Katharina Mücke, Helena Rapp, Damien B. Schlarb, Elisabeth Sommerlad, Clemens Spahr, and Dominic Zerhoch. "Immersivity: An Interdisciplinary Approach to Spaces of Immersion.” Ambiances: International Journal of Sensory Environment, Architecture and Urban Space, 2020. https://journals.openedition.org/ambiances/3233

Goldgar, Anne. Tulipmania: Money, Honor, and Knowledge in the Dutch Golden Age. Chicago, IL: The University of Chicago Press, 2017.

Goodfellow, Ian J., Jean Pouget-Abadie, Mehdi Mirza, Bing Xu, David Warde-Farley, Sherjil Ozair, Aaron Courvell and Yoshua Bengio. “Generative Adversarial Nets.” University of Montreal, 2014.

Hodge, James J. Sensations of History: Animation and New Media Art. Minneapolis, MN: University of Minnesota Press, 2019.

Hoelzl, Ingrid and Rémi Marie. Softimage: Towards a new Theory of the Digital Image. Bristol, UK: Intellect Books, 2015.

Hoelzl, Ingrid. “Image-Transaction,” in Parallax, 2020, Vol. 26, No. 1, 20-33, Networked Liminality. 
Hoy, Meredith. From Point to Pixel: A Genealogy of Digital Aesthetics. Hanover, NH:

Dartmouth College Press, 2017.

Larsen, Anders Boesen Lindbo, Søren Kaae Sønderby, Hugo Larochelle, and Ole Winther.

“Autoencoding Beyond Pixels Using a Learned Similarity Metric.” 2015.

Murray, Janet. Hamlet on the Holodeck: The Future of Narrative in Cyberspace. Cambridge,

MA: The MIT Press, 1997; updated and reissued 2016.

Reas, Casey. Making Pictures With Generative Adversarial Networks. Quebec: Anteism Books, 2019.

Rubinstein, Daniel. Fragmentation of the Photographic Image in the Digital Age. New York: Routledge, 2020.

Rubinstein, Daniel, Andy Fisher and Johnny Golding, eds. On the Verge of Photography: Imagining Beyond Representation. Birmingham, UK: ARTicle Press, 2013.

\section{Author Biography}

Holly Willis is the Chair of the Media Arts + Practice Division in USC's School of Cinematic Arts, where she teaches classes on digital media, post-cinema and feminist film. She is the author of Fast Forward: The Future(s) of the Cinematic Arts and New Digital Cinema: Reinventing the Moving Image, as well as Björk Digital, and the editor of The New Ecology of Things, a collection of essays about ubiquitous computing, and David O. Russell: Interviews. She is also the co-founder of Filmmaker Magazine, dedicated to independent film; she served as editor of RES Magazine and co-curator of RESFEST, a festival of experimental media, for several years; and she writes frequently for diverse publications about experimental film, video and new media, while also exploring experimental nonfiction and poetry. Her work has appeared in publications 
Frames Cinema Journal, Issue 19 (March 2022)

such as Film Comment, Afterimage, Los Angeles Review of Books, Variety, River Teeth and carte blanche. 